\title{
Aplicação do algoritmo MSCL com subparticionamento por múltiplas rotas
}

\author{
Clayton J. N. Lira, R.C. Almeida Jr e Daniel A. R. Chaves
}

Resumo-Por sua capacidade de fornecer recursos de forma adaptada à demanda, as Redes Ópticas Elásticas têm aparecido como uma opção de rede bastante promissora. Tais redes têm sido alvo de diversos estudos com o intuito de se apresentarem técnicas e heurísticas com a capacidade de melhor explorar seus benefícios como também encontrar soluções para seus problemas, entre eles o do roteamento e alocação de espectro. Este trabalho aborda a utilização da técnica Split Spectrum (SS) aplicada no processo de roteamento, analisando o seu desempenho em diferentes cenários e combinando com o algoritmo de atribuição de espectro MSCL. É mostrado que, combinando diferentes formas de divisão do espectro com a forma de alocação que gera a menor perda de capacidade, pode-se encontrar uma técnica que fornece melhores resultados quando comparada com a atribuição de espectro tradicional e a sua forma clássica de divisão.

Palavras-Chave-Split Spectrum, Roteamento, alocação de espectro.

Abstract-Due to its ability to provide resources adapted according to demand, Elastic Optical Networks have emerged as a very promising network option. Such networks have been the subject of several studies in order to present techniques and heuristics with the ability to explore their benefits and also find solutions to their problems, among them the routing and spectrum assignment. This work addresses the use of the Split Spectrum (SS) technique applied in the routing process, analyzing its performance in different scenarios and combining with the MSCL spectrum assignment algorithm. It is shown that, combining different forms of spectrum division with the spectrum allocation that generates the least loss of capacity, one can find a technique that provides better results when compared to the traditional spectrum allocation and its classic form of division.

Keywords-Split spectrum, Routing, Spectrum assignment.

\section{INTRODUÇÃo}

O recente crescimento da utilização de aplicações e serviços online na área de comunicações, como streaming, armazenamento em nuvem e as vídeoconferências, muito utilizadas com o crescimento do home office, fizeram com que a demanda de tráfego nas redes crescesse exponencialmente. As redes ópticas têm sido a tecnologia preferida para suportar essa crescente demanda por largura de banda. Tais redes utilizaramse, nas últimas décadas, da tecnologia WDM, (do inglês Wavelength Division Multiplexing), capaz de incrementar a capacidade de transmissão até alguns Tbps [1]. Porém, com o advento da demanda de tráfegos cada vez maiores e mais heterogênos, o requerimento da utilização de larguras de banda fixas da tecnologia WDM tornaram-na não muito eficiente [1]. As redes ópticas elásticas (EON, do inglês Elastic Optical

Clayton J. N. Lira, Departamento de Eletrônica e Sistemas (DES), UFPE; R.C. Almeida Jr, Departamento de Eletronica e Sistemas (DES), UFPE; Daniel A. R. Chave, Escola Politécnica de Pernambuco, UPE.
Networks), então, tornaram-se uma alternativa atraente, fornecendo soluções para atender às demandas com altas taxas de transmissão e tráfegos heterogêneos. Em tais redes, o espectro de frequência óptica é dividido em pequenos slots sendo que qualquer sequência desses slots, desde que sejam contíguos e estejam disponíveis ao longo de toda uma rota, pode ser atribuída à conexão. Dessa forma, o espectro pode ser usado de forma adaptada para atender aos diferentes requisitos de largura de banda da rede, permitindo gerar canais de largura variável e adaptados ao fluxo de sinal [2].

O problema do roteamento e alocação de espectro (RSA, do inglês, Routing and Spectrum Assignment) nas redes ópticas elásticas é comumente decomposto em duas partes separadas em que a primeira procura por uma rota adequada para a requisição, enquanto a segunda procura por uma porção do espectro da rota selecionada que seja conveniente para atender os requerimentos de largura de banda. Dessa forma, trabalhos recentes apresentaram propostas para aumentar o desempenho das redes ópticas, estudando formatos de modulação e heurísticas que sejam mais eficientes.

Uma vez que o problema do RSA nas redes elásticas é do tipo NP-completo, apenas estratégias heurísticas são capazes de encontrar soluções em um tempo razoável para médias e grandes topologias [3]. Alguns autores propõem mecanismos para melhor organizar a fragmentação do espectro em EON. Entre esses mecanismos, podem-se mencionar algoritmos de desfragmentação do espectro [4], conversão de comprimento de onda [5], Multiplexagem Inversa [6], [7] e Split Spectrum (SS), também denominada concatenação virtual por alguns autores [8], [9], [10], [11]. Neste artigo, a técnica de SS será aplicada em alguns cenários para comparar os benefícios obtidos da sua combinação com algoritmos de alocação de espectro (SA, do inglês Spectrum Assignment). Mais especificamente, é proposto analisar o desempenho do SS quando aplicado em conjunto com o algoritmo MSCL [12], que é projetado para analisar, para cada requisição, o estado da rede de caminhos ópticos interferentes, a fim de encontrar a porção do espectro na rede que leva à menor perda na capacidade de alocação.

\section{Algoritmo MSCL}

O algoritmo de alocação MSCL (do inglês, Min SlotContinuity Capacity Loss) é proposto para a utilização nas EONs [12]. Esse algoritmo se baseia na escolha de um conjunto de slots que estejam disponíveis e produzam a menor perda na capacidade de alocação possível nas rotas de uma dada topologia. A perda de capacidade é calculada 
considerando-se as possíveis próximas conexões que possam surgir em rotas que interfiram na rota escolhida para atender à solicitação em questão, em que considera-se que uma rota interfe com outra quando possuem pelo menos um link em comum (são chamadas rotas interferentes). O cálculo da perda de capacidade para se alocar uma requisição que demande $n$ slots é mostrado a seguir:

$$
\mathcal{C}^{<r>}(n)=\sum_{p \in I_{r}} S^{<p>}(\psi, n)-S^{<p>}\left(\psi^{\prime}, n\right),
$$

$\psi$ e $\psi^{\prime}$ são, respectivamente, os estados da rede imediatamente antes e após uma possível alocação de espectro de uma requisição com $n$ slots recebida. Já $S^{<p>}(\psi, n)$ é a capacidade, definida como o número de formas possível para se alocar demandas com tamanho $n$ nos slots disponíveis (ou seja, livres) em uma rota $p$, com estado inicial da rede dado por $\psi$. O somatório é realizado considerando-se todas as rotas do conjunto $I_{r}$, que é formado pela rota $r$ em questão e as rotas interferentes. Essas são as rotas que, de fato, perdem capacidade na alocação do espectro em questão. A perda de capacidade total na rede deve ser calculada em todo o conjunto de possíveis demandas de tráfego de largura de banda heterogênea, fornecida por:

$$
C^{<r>}=\sum_{n} \mathcal{C}^{<r>}(n),
$$

em que $\mathcal{C}(n)$ representa a perda de capacidade para cada possível requisição de tamanho de $n$ slots em uma rota.

\section{Split SPECTRUM}

A utilização da técnica de Split Spectrum permite o aumento nas possibilidades de alocação, sendo por esse motivo alvo de recente interesse na literatura. Tal característica é observada ao se dividir o número de slots exigidos em mais de um conjunto de slots que sejam contíguos. Essa técnica utiliza como princípio os conceitos de Multiplexação Inversa (MI) e Concatenação Virtual (VCAT, do inglês Virtual Concatenation) em um roteamento de ponta a ponta.

A utilização dessa técnica acarreta certas vantagens, como o uso de taxas mais baixas do que a da demanda original. Este procedimento permite que requisições sejam mais facilmente acomodadas, permitindo a utilização de buracos menores (um buraco é o conjunto de slots que sejam contíguos e estejam disponíveis) [8]. Quando comparada com outras técnicas, como por exemplo a de Multiplexação Inversa que permite a divisão da requisição em nós intermediários [6], o fato do SS particionar requisições apenas nos nós de origem pode proporcionar uma maior eficiência energética, fornecendo, dessa forma, uma solução completa para a camada óptica e também reduzindo a complexidade dos nós intermediários, graças à transparência nos protocolos de comunicação e taxas de dados [8]. No entanto, a aplicação da técnica de SS pode promover a inserção de requisitos adicionais na rede, principalmente nos elementos físicos e plano de controle. A sua utilização também pode promover pequenos atrasos adicionais no estabelecimento do caminho óptico, podendo necessitar de um armazenamento inicial do tráfego [9].

\section{Analis ando o Desempenho do Split Spectrum JUNTAMENTE COM O MSCL}

Considerando-se a possibilidade de uso da técnica de SS na rede, a sua utilização permite um aumento nas possibilidades de alocação das chamadas, uma vez que existem diversas formas de se dividir uma certa demanda, e diferentes posições do espectro podem ser escolhidas para a sua alocação. Um próximo passo a ser considerado a seguir é o da análise da melhor maneira de se dividir a requisição e de qual a melhor porção do espectro da rede para se alocá-la. Para tanto, este trabalho considerará a utilização do algoritmo MSCL, que é capaz de analisar o estado da rede através dos cálculos de perda de capacidade de alocação. Neste artigo adapta-se o MSCL de forma que seja possivel escolher a menor perda de capacidade explorando os seguintes aspectos: a escolha da melhor forma de dividir uma solicitação; a escolha dos buracos nos quais as partes solicitadas serão inseridas; o grupo de slots usados dentro de cada buraco. Para cada parte da requisição dividida, calcula-se a perda de capacidade, testando-se suas possíveis alocações nos slots da rede que estejam disponíveis. A perda de capacidade combinada é obtida somando-se as perdas individuais para cada parte da requisição dividida. A possibilidade que retornar a menor perda de capacidade será, então, a escolhida. Considerando-se a existência de diferentes modelos de roteamento, pode-se citar dois dos mais utilizados na literatura como sendo o roteamento fixo, em que é escolhida uma rota que conecte os nós de origem e destino e que seja capaz de atender à requisição em questão, e o roteamento fixo-alternativo em que é escolhido um conjunto de rotas que conectam os nós origem e destino. Nesse roteamento, quando a rota principal não possui recursos suficientes para atender a demanda, a próxima rota do conjunto será escolhida para a alocação e esse processo continua até que a requisição seja alocada no espectro de alguma rota ou nenhuma rota do conjunto escolhido possa atender a essa requisição, que será então bloqueada [18].

Ao se adaptar a técnica de SS para a utilização em EONs, duas são as abordagens possíveis de serem avaliadas. $\mathrm{Na}$ primeira abordagem, a requisição dividida pode ser roteada considerando-se um único caminho. A essa abordagem dá-se o nome SPA (do inglês Single Path Approach). A segunda abordagem possível, chamada MPA (do inglês Multi Path Approach), considera o roteamento das partes provenientes do processo de divisão pelo SS através de rotas distintas. Esse procedimento é capaz de obter um melhor desempenho em relação à probabilidade de bloqueio das requisições, apesar de possuir um processamento mais complexo [11], [13].

Para a aplicação da técnica de Split Spectrum considerase a utilização de transponders capazes de realizar tal procedimento, formando subportadoras internas independentes. Esses transponders são conhecidos como SBVT (do inglês, Sliceable Bandwidth Variable Transponders). Esses transponders possuem uma capacidade conhecida como sliceability que permite com que cada dispositivo possa ser internamente separado em vários sub-transponders, sendo que cada um deles tem a capacidade de estabelecer um caminho completo entre um par de nós origem-destino [14], [15]. As duas principais 
tecnologias adotadas na utilização desses transponders são a ML-SBVT (do inglês, Multi Laser Sliceable Bandwidth Variable Transponder) e MW-SBVT (do inglês, Multi Wavelength Sliceable Bandwidth Variable Transponder). A primeira delas utiliza vários lasers para a transmissão em um transponder, enquanto a segunda utiliza vários comprimentos de onda sendo transmitidos em um único laser. Considerando-se a utilização dessas duas tecnologias, a MW-SBVT possui a restrição de necessitar que as subportadoras devem ser alocadas de forma contígua ou muito próximas umas das outras, com um espaçamento máximo de $50 \mathrm{GHz}$ [14]. Já a tecnologia ML-SBVT permite uma maior liberdade de escolha entre as subportadoras, tendo em vista que cada uma delas utiliza um laser diferente, permitindo assim que as partes resultantes do processo de SS possam ser alocadas em qualquer faixa de espectro dentro das rotas, exceto nos slots que possuem o mesmo índice dentro das rotas consideradas como alternativas para o roteamento. Por conta dessas vantagens, apesar do custo da utilização de vários lasers para a transmissão, a tecnologia ML-SBVT é a mais comumente utilizada nos trabalhos que consideram a utilização da técnica de SS e será a tecnologia adotada no presente trabalho [15], [16], [17].

\section{A. Abordagens utilizadas}

Esse trabalho considerará duas etapas para a análise do desempenho da combinação da técnica de SS com o algoritmo de alocação MSCL, etapas essas que são adotadas dentro da mesma heurística. A primeira dessas etapas considera que o roteamento das partes resultantes do processo de divisão se dá através de uma única rota, utilizando a abordagem conhecida como SPA (do inglês, Single Path Approach). Já a segunda etapa da heurística considera a abordagem conhecida como MPA (do inglês Multi Path Approach), abordagem essa que considera que as partes resultantes do processo de dvisão podem ser transmitidas através de rotas distintas [11]. Ao se considerar a transmissão através de múltiplas rotas, uma limitação a ser considerada é o atraso entre a chegada das partes da requisição particionada, provenientes de rotas distintas [13]. Para mitigar tal limitação, esse trabalho considera que as rotas adotadas no roteamento das partes provenientes do processo de SS não podem possuir mais de um hop de diferença entre si, para que dessa forma o delay entre o recebimento das partes da requisição se esteabeleça dentro de limites aceitáveis, não prejudicando o processamento do sinal recebido. Em ambas etapas será utilizado o algoritmo de alocação MSCL que, através de sua capacidade de análise do estado da rede, auxiliará na escolha da melhor forma de se dividir a requisição e de qual as melhores posições no espectro para se introduzir a requisição, tendo sido ela dividida ou não.

No presente trabalho será considerada a combinação da técnica de SS com o algoritmo MSCL apenas. A escolha desse algoritmo se deu pela habilidade do MSCL em analisar o estado da rede, fazendo com que ele se beneficie do processo de divisão da requisição original que proporciona um aumento nas possibilidades de alocação testadas pelo algoritmo, fazendo com que ele obtenha melhores resultados quando comparado a outros algoritmos, como o First-Fit, por exemplo [13]. A seguir é apresentada a heurística, que será chamada MPA-MSCL, (do inglês, Multi Path Approach with Min Slot-Continuity Capacity Loss), já que combina a utilização do algoritmo MSCL com a abordagem MPA de roteamento através de múltiplas rotas utilizando a técnica de Split Spectrum com o algoritmo MSCL. A heurística será dividida em duas etapas, sendo que a primeira é focada no roteamento através de uma única rota, enquanto a segunda foca na abordagem MPA. Como dito anteriormente, as duas etapas compõem a mesma heurística e se complementam.

1) Etapa SPA: A primeira etapa da heurística considera a abordagem que trata do roteamento através de uma única rota, considerando que a requisição em questão pode ou não ser dividida. Para se evitar o aumento da complexidade promovido pela adoção da técnica de SS, sempre será dada prioridade à alocação da requisição completa, sem que esta seja dividida. Considerando que a requisição necessitará ser particionada, ainda para se minimizar o custos do aumento da complexidade, se dará prioridade à adoção da menor quantidade de divisões possíveis na requisição. Dessa forma, a alocação da heurística considera os seguintes passos nessa primeira etapa:

- Tenta-se alocar a requisição na rota principal encontrada pelo algoritmo de roteamento $\left(r_{1}\right)$ ou em alguma das rotas alternativas, sem considerar a divisão através da adoção da técnica de Split Spectrum. Para diminuir a complexidade, são consideradas até duas rotas alternativas, fazendo um total de três rotas que podem ser utilizadas para o roteamento da requisição, sendo essas as rotas $r_{1}, r_{2}$ e $r_{3}$, ordenadas da mais curta para a mais longa.

- Se a etapa anterior não for suficiente, a técnica de SS será utilizada e a requisição será particionada em duas ou três outras menores. Será dada prioridade à alocação nas rotas mais curtas, portando inicialmente serão testadas todas as possibilidades de alocação na rota principal, $r_{1}$, com a requisição original sendo dividida em 2 ou em 3 partes.

- Caso a requisição não consiga ser alocada mesmo sendo dividida em três partes separadas na rota principal, serão utilizadas as outras rotas alternativas $r_{2}$ e $r_{3}$, nessa ordem, seguindo os mesmos passos do ítem anterior. A escolha dos slots utilizados na alocação no espectro da rede é feita através do algoritmo MSCL.

Nessa primeira etapa o algoritmo irá testar todas as possibilidades de alocação da requisição nas rotas em questão. Ao se considerar a divisão da requisição original, serão testadas também todas as possibilidades de divisão, escolhendo a que proporcionar a menor perda de capacidade de alocação combinada (somando as perdas de capacidade individuais de cada parte da requisição). Caso essa etapa não seja suficiente para alocar a reqisição, será considerado o roteamento através de rotas distintas descrito a seguir.

2) Etapa MPA: Observa-se na etapa anterior que, até esse ponto, a heurística considera a utilização de apenas uma rota $r$, que pode alocar a requisição inteira ou dividida em 2 ou 3 partes. Um próximo passo seria aproveitar mais a capacidade da técnica de Split Spectrum, utilizando a abordagem MPA e permitindo o roteamento através de rotas distintas de forma simultânea. Essa etapa será então adotada quando a SPA 
não for suficiente para alocar a requisição, considerando o roteamento através de até 3 rotas simultaneamente, desde que elas não possuam mais de um hop de diferença entre si. Os passos adotados na implementação dessa etapa são descritos a seguir:

- Considerando o roteamento através de múltiplas rotas, continua-se dando prioridade à alocação na rota principal ou nas primeiras rotas alternativas (rotas mais curtas). Para tanto, será alocada a maior fração possível da requisição original na rota principal $\left(r_{1}\right)$, sendo que o restante da requisição que não puder ser alocada nessa rota será transmitida através da primeira rota alternativa $\left(r_{2}\right)$. Caso a rota alternativa $r_{2}$ em questão não possua recursos suficientes para alocar a parte restante do processo de divisão como um todo, será considerada a aplicação da técnica de Split Spectrum apenas nessa parte da requisição, dividindo-a em 2 outras partes menores, que tentarão ser alocadas na mesma rota alternativa $\left(r_{2}\right)$, em buracos separados.

- Caso a primeira rota alternativa $\left(r_{2}\right)$ não possua recursos suficientes para alocar o restante da requisição, mesmo particionando, ela deverá receber a maior fração possível na requisição restante. A fração que sobrar após a alocação em $r_{2}$ será transmitida através da próxima rota alternativa, $r_{3}$, sendo que a requisição será bloqueada se não conseguir ser alocada seguindo todos esses passos.

Percebe-se que a heurística busca esgotar primeiramente os recursos das rotas mais curtas. Para se minimizar o aumento do custo computacional da heurística, busca-se alocar a requisição sem que essa seja dividida, seja na rota principal ou nas alternativas. Ao se considerar a utilização da técnica de SS, a heurística testa primeiramente todas as possibilidades de alocação na rota $r_{1}$, testando a alocação da requisição dividida em 3 partes na rota $r_{1}$ antes de testar a alocação da requisição dividida em 2 partes mas na rota $r_{2}$. A adoção desse modo de teste deu-se ao fato desse trabalho não considerar a quantidade de transponders disponíveis para a alocação nos nós, o que torna indiferente a adoção de 1 ou 2 divisões na requisição original quando as partes forem transmitidas através da mesma rota. Como forma de resumir e simplificar os passos descritos anteriormente, é mostrado um diagrama descrevendo os passos da heurística na Fig. 1.

\section{ANALISANDO OS RESULTADOS OBTIDOS}

Foram realizadas simulações considerando a transmissão através de múltiplas rotas, utilizando um roteamento fixoalternativo, em que um conjunto de rotas entre um par origem destino é criado para atender as demandas e os elementos desse conjunto de rotas seguem uma ordem pré-estabelecida. Nesse tipo de roteamento, a rota que primeiro possuir recursos espectrais suficientes para atender a requisição é selecionada para a alocação do espectro [19]. Foi observado que, à medida que aumenta-se quantidade de rotas alternativas, diminui-se a probabilidade de bloqueio das requisições até um ponto em que o aumento na quantidade de rotas não exerce mais muita influência na redução desse bloqueio. Esse dado foi observado através de simulações feitas para a topologia de rede

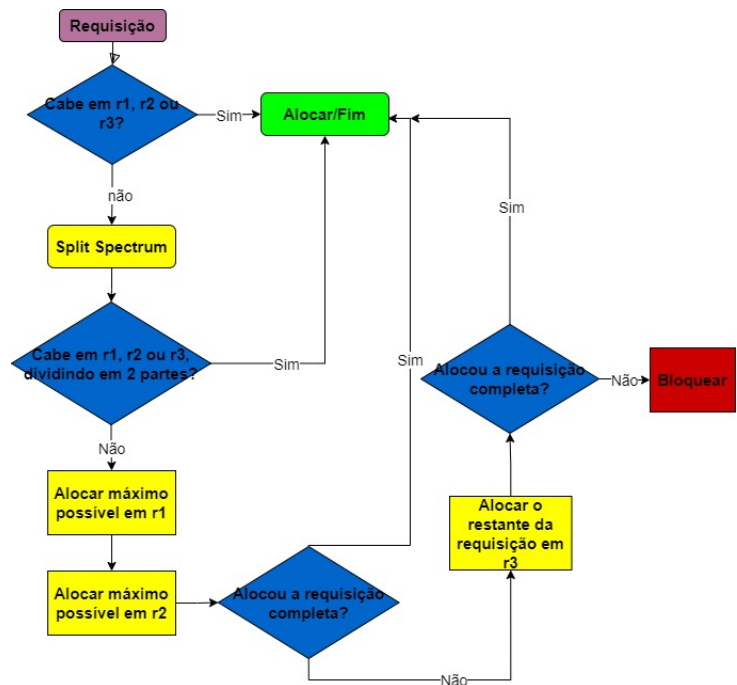

Fig. 1. Diagrama simplificado da heurística proposta

NSFNet com a utilização do algoritmo MSCL, aumentandose gradualmente a quantidade de rotas alternativas de 1 até 6 rotas. As simulações utilizadas nesse trabalho não consideram limitações da camada física e quantidade de transponders em cada nó, considerando a utilização de apenas um formato de modulação em todas as rotas. O resultado é mostrado na Tabela I a seguir.

TABELA I

REDUÇÃO NA PROBABILIDADE DE BLOQUEIO COM O AUMENTO NA QUANTIDADE DE ROTAS ALTERNATIVAS.

\begin{tabular}{|c||c|c|c|c|c|}
\hline & 2 rotas & 3 rotas & 4 rotas & 5 rotas & 6 rotas \\
\hline \hline MSCL & $29 \%$ & $15 \%$ & $9 \%$ & $5 \%$ & $2 \%$ \\
\hline MSCL+SS & $66 \%$ & $34 \%$ & $26 \%$ & $12 \%$ & $12 \%$ \\
\hline
\end{tabular}

Percebe-se através da tabela que a redução na probabilidade de bloqueio variou entre $29 \%$, quando se utiliza 2 rotas alternativas, até $5 \%$ quando se utiliza 5 rotas alternativas e $2 \%$ com 6 rotas. A partir desse ponto não percebe-se benefício com o aumento da quantidade de rotas alternativas. Porém, considerando a utilização da forma mais básica de Split Spectrum em que a requisição original é dividida em duas outras menores, permitindo a alocação das partes resultantes do processo de divisão apenas dentro da própria rota (SPA), observa-se uma redução na probabilidade de bloqueio ainda considerável com o aumento da quantidade de rotas alternativas, variando entre $66 \%$ ao se considerar 2 rotas e $12 \%$ com 6 rotas alternativas. Dessa forma, o benefício da utilização da tecnologia de SS já justifica o seu uso mesmo ao se considerar sua forma mais simples, com roteamento através de uma única rota. Esse estudo inicial foi realizado com o intuito de se entender o desempenho da aplicação da técninca de SS nos cenários com várias rotas alternativas, não sendo aplicado na heurística que considera a abordagem MPA pelo fato do aumento demasiado na quantidade de rotas alternativas ferir a limitação da diferença máxima de 1 hop entre as rotas. A Topologia NSFNet com 14 nós e 42 links foi utilizada, com um 
total de 64 slot por link. Para cada simulação, foram realizadas $10^{6}$ requisições, com processo de chamada poissoniano com taxa de desativação de chamadas por segundo igual a 1, fazendo com que a carga represente diretamente a taxa de chegada de requisições por segundo, sendo a duração das requisições distribuídas exponencialmente. Foi considerado um tráfego com solicitações assumindo larguras de banda variando uniformemente entre 2 e 6 slots, sendo os pares de nós de origem e destino escolhidos aleatoriamente e com igual probabilidade. A seguir são apresentados os valores de probabilidade alcançados para a heurística proposta.

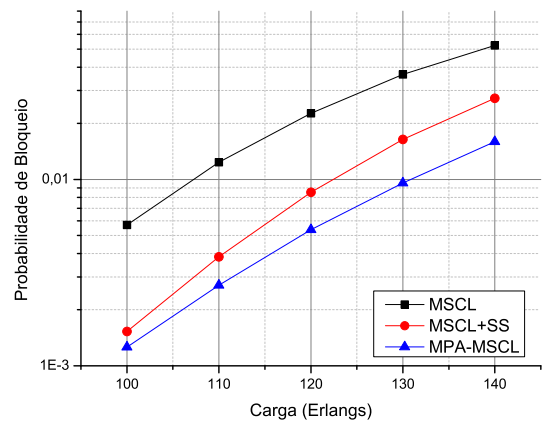

Fig. 2. Simulação mostrando a curvas de Probabilidade de Bloqueio para as heurísticas MSCL, MSCL com SS dentro de uma mesma rota e MPA-MSCL.

Observa-se a partir da Fig. 2 que a utilização da técnica de SS é capaz de reduzir drasticamente a probabilidade de bloqueio, mesmo quando combinada com o algorítmo MSCL que já é capaz de apresentar um bom desempenho nesse quesito. A curva MSCL+SS, em vermelho, representa a utilização da técninca de SS combinada com o algoritmo MSCL, porém sem considerar a possibilidade de roteamento através de múltiplas rotas, sendo que a divisão e transmissão da requisição acontece através da rota principal ou de alguma das rotas alternativas. A curva MPA-MSCL, em azul, mostra a redução na probabilidade de bloqueio obtida pela heurística apresentada, especialmente em cargas mais elevadas em que a rede encontra maior dificuldade em alocar as requisições pela diminuição dos recursos disponíveis.

\section{CONCLUSÕES}

A técnica de Split Spectrum se beneficia da sua combinação com o algoritmo MSCL, que permite a análise do estado da rede ao se alocar as requisições. Essa combinação também gera benefícios para esse algoritmo de alocação, já que a divisão da requisição em outras menores proporciona um aumento nas possibilidades de alocação analisadas. Os resultados mostram um aumento significativo no desempenho da técnica combinada quando se considera o roteamento através de múltiplas rotas, fornecendo uma redução na probabilidade de bloqueio das requisições e dando margem a utilização de mais rotas alternativas. Porém, a diferença entre os comprimentos das rotas alternativas e o tempo de simulação podem ser fatores limitantes ao se aumentar o número de rotas e quantidade de divisões na requisição original.

\section{AGRADECIMENTOS}

Os autores agradecem o apoio institucional da Universidade Federal de Pernambuco (UFPE) e Universidade de Pernam- buco (UPE) e o apoio financeiro da Capes e FACEPE.

\section{REFERÊNCIAS}

[1] Talebi, Sahar Alam, Furqan Katib, Iyad Khamis, Mohammed Khalifah, Reda \& Rouskas, G.N.. "Spectrum management techniques for elastic optical networks: A survey". Optical Switching and Networking, 2014.

[2] Gerstel, O., Jinno, M., Lord, A., and Yoo, S.J.B.: "Elastic optical networking: a new dawn for the optical layer?,"IEEE Commun. Mag., 2012, 50, pp. 12-20.

[3] B. C. Chatterjee, N. Sarma and E. Oki, "Routing and Spectrum Allocation in Elastic Optical Networks: A Tutorial,"in IEEE Communications Surveys Tutorials, vol. 17, no. 3, pp. 1776-1800, thirdquarter, 2015.

[4] M. Zhang, W. Shi, L. Gong, W. Lu, and Z. Zhu, "Bandwidth Defragmentation in Dynamic Elastic Optical Networks with Minimum Traffic Disruptions, "IEEE International Conference on Communication (ICC), Jun. 2013.

[5] X. Wang, M. Brandt-Pearce, and S. Subramaniam, "Impact of Wavelength and Modulation Conversion on Translucent Elastic Optical Networks Using MILP,"J. Opt. Commun. Netw. 7, 644-655 (2015).

[6] Y. Xu, J. Li, P. Zhu, B. Guo, Y. Chen, Y. Zhong, Y. Wang, Z. Chen, and Y. He, "Demonstration of All-optical Inverse Multiplexing in Elastic Optical Networks,"in Optical Fiber Communication Conference, OSA Technical Digest (online) (Optical Society of America, 2014), paper Th1E.6.

[7] Bingli Guo, Yingying Xu, Paikun Zhu, Yucheng Zhong, Yuanxiang Chen, Juhao Li, Zhangyuan Chen, and Yongqi He, "Multicasting based optical inverse multiplexing in elastic optical network,"Opt. Express 22, 15133-15142 (2014)

[8] Ming Xia, R. Proietti, Stefan Dahlfort, and S. J. B. Yoo, "Split spectrum: a multi-channel approach to elastic optical networking,"Opt. Express 20, 29143-29148 (2012).

[9] S. Dahlfort, M. Xia, R. Proietti, and S. J. B. Yoo, "Split Spectrum Approach to Elastic Optical Networking,"in European Conference and Exhibition on Optical Communication, ECOC 2012.

[10] Y. Zhang, Y. Zhang, S. K. Bose and G. Shen, "Migration From Fixed to Flexible Grid Optical Networks With Sub-Band Virtual Concatenation,"in Journal of Lightwave Technology, vol. 35, no. 10, pp. 17521765, 15 May15, 2017.

[11] Albert Pagès, Jordi Perelló, Salvatore Spadaro, and Jaume Comellas, "Optimal Route, Spectrum, and Modulation Level Assignment in SplitSpectrum-Enabled Dynamic Elastic Optical Networks,"J. Opt. Commun. Netw. 6, 114-126 (2014).

[12] R.C Almeida Jr., A.F. Santos, K.D.R. Assis, J.F. Martins-Filho, and H. Waldman, "Slot assignment strategy to reduce loss of capacity of contiguous-slot path requests in flexible grid optical networks". Electronics Letters, v. 49, p. 359-361, 2013.

[13] LIRA, C. J. N.; ALMEIDA, RAUL C.; CHAVES, D. A. R. Estratégias de RSA Utilizando Split Spectrum e o algoritmo MSCL em EONs In: XXXVIII Simpósio Brasileiro de Telecomunicações e Processamento de Sinais - SBrT 2020, 2020, Florianópolis (virtual).

[14] M. Dallaglio, A. Giorgetti, N. Sambo, L. Velasco e P. Castoldi, "Routing, Spectrum, and Transponder Assignment in Elastic Optical Networks,"Journal of Lightwave Technology, vol. 33, n. 22, pp. 4648 - 4658, 2015.

[15] N. Sambo, G. Meloni, F. Paolucci, M. Imran, F. Fresi, F. Cugini, P. Castoldi e L. Poti, "First demonstration of SDN-controlled SBVT based on multi-wavelength source with programmable and asymmetric channel spacing,"em The European Conference on Optical Communication (ECOC), Cannes, France, 2014.

[16] X. Wang, G. Shen, Z. Zhu e X. Fu, "Benefits of Sub-band Virtual Concatenation for Enhancing Availability of Elastic Optical Networks,"JOURNAL OF LIGHTWAVE TECHNOLOGY, vol. 34, n. 4, 2016.

[17] X. Yuan e G. Shen, "How Much Can Sub-band Virtual Concatenation (VCAT) Help Static Routing and Spectrum Assignment in Elastic Optical Networks?,"em IEEE International Conference on Communication Systems, 2014

[18] Zang, H., Jue, J. P., Mukherjee, B. (2000). "A review of routing and wavelength assignment approaches for wavelength-routed optical wdm networks". Optical Networks Magazine.

[19] Murthy, C. S. R.; Gurusamy, M. Wdm optical networks - concepts, design and algorithms. Prentice Hall PTR, 2002. 